\title{
A Spatial Interaction Model for Deriving Joint Space Maps of Bundle Compositions and Market Segments from Pick-Any/J Data: An Application to New Product Options
}

\author{
WAYNE S. DESARBO \\ School of Business Administration, University of Michigan, Ann Arbor, MI 48109 \\ VENKATRAM RAMASWAMY \\ School of Business Administration, University of Michigan, Ann Arbor, MI 48109 \\ MICHEL WEDEL \\ Faculty of Economics, University of Groningen, Netherlands \\ TAMMO BIJMOLT \\ Department of Marketing, Tilburg University, Netherlands
}

\begin{abstract}
We propose an approach for deriving joint space maps of bundle compositions and market segments from threeway (e.g. , consumers $\times$ product options/benefits/features $\times$ usage situations/scenarios/time periods) pick-any/J data. The proposed latent structure multidimensional scaling procedure simultaneously extracts market segment and product option positions in a joint space map such that the closer a product option is to a particlar segment, the higher the likelihood of its being chosen by that segment. A segment-level threshold parameter is estimated that spatially delineates the bundle of product options that are predicted to be chosen by each segment. Estimates of the probability of each consumer belonging to the derived segments are simultaneously obtained. Explicit treatment of product and consumer characteristics are allowed via optional model reparameterizations of the product option locations and segment memberships. We illustrate the use of the proposed approach using an actual commercial application involving pick-any/J data gathered by a major hi-tech firm for some 23 advanced technological options for new automobiles.
\end{abstract}

\section{Introduction}

A major high technology firm recently conducted extensive consumer interviews for investigating the desirability of a number of innovative, advanced technological options for automobiles (adaptive cruise control, visibility lighting, adaptive head-up display, all weather windows, and so on) that it was considering for "premarketing" to several major automobile manufacturers. One of the main objectives of this study was to understand the "complementarity" among these various options as judged by consumers and to delineate market segments and associated bundles (or subsets) of options desired by each segment of the 
market. In personal interviews with appropriately screened respondents, each of the options were described with suggested prices. Assuming availability, respondents were asked to choose those options they would definitely consider purchasing jointly (as a bundle) with their next vehicle. The data for the entire sample can be summarized via a two-way (consumers $x$ new product options) matrix, where each respondent's bundle composition is represented by a vector of ones and zeroes, with the ones denoting the chosen options. Had the same data been collected for different usage situations (Jedidi and DeSarbo, 1991), then the corresponding binary data would have been organized in a three-way array (situation $X$ consumers $\times$ new product options). Such binary data are referred to as "pickany/J" data and are easy to collect and widely used in market research (Green, Schaffer, and Patterson, 1988).

Various approaches have been advanced for the spatial analysis of pick-any/J data. Correspondence analysis and other data analytic procedures entail the fitting of alternative spatial representations to input data matrices (e.g., Hoffman and Franke, 1986; Holbrook, Moore, and Winer, 1982; Lebart, Morineau, and Warwick, 1984; Nishisato, 1980) so as to derive a "joint space" of respondents and objects (new product options in the above example). Such procedures do not posit any underlying theory of choice. In contrast, the procedure of DeSarbo and Hoffman (1987) is based on (indirect) utility theory (McFadden 1976) and utilizes an unfolding representation. However, this procedure, like traditional spatial models, is less suited for large samples of the size normally encountered in marketing research. The number of parameters estimated can become excessive, the joint space representations become saturated with ideal points, and interpretation can be virtually impossible with large samples. In addition, marketers are often interested in segmenting consumers and targeting specific market segments (cf. Wind 1978), since segmentation and positioning are at the heart of any marketing strategy.

Given the twin objectives of spatial analysis and market segmentation, it is common to engage in a two-step approach of initially using a data analytic procedure like correspondence analysis for spatial analysis followed by a cluster analysis of the individual coordinates to form segments. However, this two-step traditional approach can be problematic as the cluster and spatial analyses typically optimize different objective functions relating to different aspects of the data. There is little theory to guide the selection of the clustering procedure and different clustering algorithms will typically produce different market segmentation results (cf. DeSarbo, Wedel, Vriens, and Ramaswamy, 1992). Further, several of the smaller dimensions in spatial analysis that are often discarded contain much of the clustering information concerning the objects to be clustered (see DeSarbo, Ramaswamy, and Lenk, 1993). Recognizing these limitations, recent "latent structure" spatial approaches (see DeSarbo, Manrai, and Manrai, 1994 for a review) attempt to perform spatial analysis (i.e., MDS) and market segmentation (i.e., cluster analysis) simultaneously, estimating segment-specific, rather than individual-specific, ideal points or vectors, thereby reducing the number of parameters to be estimated and deriving representations that are more managerially interpretable.

In this paper, we present a generalized, latent structure stochastic unfolding model for the segment-level analysis of three-way, pick-any/J data. The proposed model is a singlestep procedure that simultaneously derives a joint space of market segments and products, thereby overcoming the problem of saturation of ideal points and/or eliminating the need for a subsequent cluster analysis to form segments. Further, it overcomes another limitation 
of the DeSarbo and Hoffman (1987) approach in that it can accommodate three-way, pickany/J data (e.g., DeSarbo, Lehmann, Gupta, Holbrook, and Havlena, 1987; Jedidi and DeSarbo, 1991; Stefflre, 1971) such as respondents $\times$ product features/benefits/options $x$ consumption situations/scenarios/time periods. Situation-specific weights for the dimensions of the joint space are estimated given such three-way, pick-any/J data.

\section{The proposed model}

Let

$i=1, \ldots, I$ consumers,

$j=1, \ldots, J$ product options/features/benefits,

$n=1, \ldots, N$ situations/scenarios/time periods,

$s=1, \ldots, S$ market segments,

$t=1, \ldots, T$ dimensions,

$k=1, \ldots, K$ product characteristics (e.g., physical aspects, functionality, price),

$m=1, \ldots, M$ consumer characteristics (e.g., demographic and/or psychographic variables),

$\delta_{i j n}=1$ if consumer $\mathrm{i}$ considers/chooses option $\mathrm{j}$ in time $\mathrm{n}$, or 0 otherwise.

We define a latent, unobservable "disutility" variable $D_{s j n}$ for option $j$ in situation (generically used here) $n$ for segment $s$ :

$$
D_{s j n}=\sum_{t=1}^{T} w_{n t}\left(a_{s t}-b_{j t}\right)^{2}+\epsilon_{s j n}
$$

where $\epsilon_{s j n}$ is a stochastic error component that is assumed to be i.i.d. normally distributed with a mean of zero and variance $\theta_{s}$. Equation 1 denotes that $D_{s j n}$, the latent disutility for option $j$ within segment $s$ in situation $n$, can be represented by a weighted unfolding model (DeSarbo and Carroll, 1985), involving the ideal point for segment $s\left(\mathbf{A}_{s}\right)$, option $j$ 's coordinates $\left(B_{j}\right)$, and the salience (nonnegative weight), $w_{n t}$, for dimension $t$ in situation $n$. If the data are not gathered over different situations as in two-way analyses, $w_{n t}=1$ for all $t$, and the simple unfolding model results (DeSarbo, Manrai, and Manrai, 1994). The parameter $w_{n t}$ represents the differential salience of the dimensions in different situations.

We assume that for any consumer $i$ belonging to segment $s$, product option $j$ would be considered/chosen if it exceeds the segment's (unknown) "threshold" utility (or alternatively, if it does not exceed the segment's threshold disutility, denoted as $c_{s}$ ). Therefore, if the threshold value $c_{s}$ for segment $s$ is greater than or equal to the value of the unobservable latent variable, segment $s$ will include the product option in question in its "bundle of options"; if the threshold value is less than the value of the unobservable latent variable, segment $s$ will not include the product option (see DeSarbo and Hoffman, 1987, for the psychological and economic rationale for thresholds in such random disutility models). In terms of the ideal point representation, product option $j$ is included in the bundle of segment $s$ when it is close to the segment's ideal notion of a product option. 
Thus, the probability of consumer $i$ choosing product option $j$ in situation $n$, conditional upon membership in segment $s, P_{i j n \mid s}$, can be derived as

$$
\begin{aligned}
P_{s}\left(\delta_{i j n}=1\right) & =P\left(D_{s j n} \leq c_{s}\right) \\
& =\Phi\left[\frac{c_{s}-\sum_{t=1}^{T} w_{n t}\left(a_{s t}-b_{j t}\right)^{2}}{\theta_{s}}\right],
\end{aligned}
$$

where $\Phi(\cdot)$ is the cumulative normal distribution function. The derived joint space map simultaneously portrays the product options and the ideal points of the derived market segments, as well as the respective bundles of options preferred by each segment (vis à vis $c_{s}$ ).

Assuming that consumer $i$ has an unknown prior probability $\lambda_{s}$ of belonging to segment $s$, the unconditional likelihood for consumer $i$ can be expressed as a finite mixture of the $S$ conditional likelihood functions (Titterington, Smith, and Makov, 1985):

$$
L_{i}=\sum_{s=1}^{S} \lambda_{s} L_{i \mid s}
$$

where the prior probabilities obey the constraints

$$
\sum_{s=1}^{S} \lambda_{s}=1, \text { and } 0<\lambda_{s}<1
$$

Note that unlike conditional logit latent structure models (cf. Kamakura, Wedel, and Agrawal, 1994), the proposed model need not have the constraint that $\Sigma_{j} P_{i j n \mid s}=1$ given that consumers' choices across the $J$ alternatives are being modeled as being independent of each other within a segment. Conditional independence is assumed in such latent structure models, and it is the joint space representation that portrays the complementarity across the $J$ product options. ${ }^{1}$ Hence, the model is well suited for describing bundle compositions that reflect the complementarity among product options within segments of consumers. The use of pick-any/J data does not impose any structural dependencies on the number or format of the choices and non-choices of respondents.

Given (binary) pick-any/J binary data for consumer $i$ over $N$ situations, we can write the conditional likelihood function, $L_{i \mid s}$ as

$L_{i \mid s}=\prod_{n=1}^{N} \prod_{j=1}^{J} \Phi\left[\frac{c_{s}-\sum_{t=1}^{T} w_{n t}\left(a_{s t}-b_{j t}\right)^{2}}{\theta_{s}}\right]^{\delta_{i j n}} \cdot\left[1-\Phi\left[\frac{c_{s}-\sum_{t=1}^{T} w_{n t}\left(a_{s t}-b_{j t}\right)^{2}}{\theta_{s}}\right]\right]^{1-\delta_{i j n}}$, 
so that the unconditional likelihood function $L_{i}$ is

$$
L_{i}=\sum_{s=1}^{S} \lambda_{s} \prod_{n=1}^{N} \prod_{j=1}^{J} \Phi\left(f_{s j n}\right)^{\delta_{i j n}} \cdot\left(1-\Phi\left(f_{s j n}\right)\right)^{1-\delta_{i j n}}
$$

where

$$
f_{s j n}=\frac{c_{s}-\sum_{t=1}^{T} w_{n t}\left(a_{s t}-b_{j t}\right)^{2}}{\theta_{s}}
$$

The complete log likelihood function for the entire sample of three-way (i.e., $I$ consumers $\times J$ product options $\times N$ situations) pick-any/J data can therefore be expressed as

$$
\ln L=\sum_{i=1}^{I} \ln \left[\sum_{s=1}^{S} \lambda_{s} \prod_{n=1}^{N} \prod_{j=1}^{I} \Phi\left(f_{s j n}\right)^{\delta_{i j n}} \cdot\left(1-\Phi\left(f_{s j n}\right)\right)^{1-\delta_{i j n}}\right]
$$

As such, the proposed latent structure MDS methodology can be viewed as a generalization of the Böckenholt and Böckenholt (1991) and Wedel and DeSarbo (1995) procedures to accommodate three-way pick-any/J data, as well as to allow for various reparameterized model options discussed subsequently.

Conditional on current parameter estimates, one can estimate (simultaneously within any iterate) the posterior probabilities of segment membership as

$$
\hat{Q}_{i s}=\frac{\hat{\lambda_{s}} \prod_{n=1}^{N} \prod_{i=1}^{J} \Phi\left(\hat{f}_{s j n}\right)^{\delta_{i j n}} \cdot\left(1-\Phi\left(\hat{f}_{s j n}\right)\right)^{1-\delta_{i j n}}}{\sum_{s=1}^{S} \hat{\lambda}_{s} \prod_{n=1}^{N} \prod_{j=1}^{J} \Phi\left(\hat{f}_{s j n}\right)^{\delta_{i j n}} \cdot\left(1-\Phi\left(\hat{f_{s j n}}\right)\right)^{1-\delta_{i j n}}},
$$

where " " denotes values based on the estimated parameters (the estimation process is to be discussed shortly) and $\hat{Q}_{i s}$ is the estimated posterior probability of membership into segment $s$ for consumer $i$.

\subsection{Reparameterized model options}

The proposed model can be generalized to incorporate additional data in the form of respondent background variables to aid in targeting chosen segments and product characteristics to aid in the interpretation of the derived dimensions (Wedel and DeSarbo, 1995). The consumer background data can be accommodated by reparameterizing the prior probability of membership into segment $s$ for consumer $i$ as (Dayton and MacReady, 1988) 


$$
\ln \left[\frac{\lambda_{i s}}{\lambda_{i S}}\right]=\sum_{m=1}^{M} z_{i m} \gamma_{m s}
$$

where $z_{i m}$ is the value of characteristic $m$ for consumer $i$ and $\gamma_{m s}$ is the impact coefficient for characteristic $m$ for segment $s$. The product coordinates can also be reparameterized as a linear function of product characteristics. Hence, if product attribute data are available, $b_{j t}$ can be reparameterized as

$$
b_{j t}=\sum_{k} x_{j k} \alpha_{k t}
$$

where $x_{j k}$ is the value of characteristic $k$ for option $j$ and $\alpha_{k t}$ is the impact of feature $k$ on dimension $t$. The location of a product is thus modeled as a direct function of respective characteristics (cf. DeSarbo and Rao, 1986), thereby providing a link between a product's objectively quantifiable characteristics and its perceived spatial location in the derived joint map.

\subsection{Estimation issues}

The goal of the estimation procedure is to maximize the log likelihood function (8) with respect to the parameters: $\Lambda=\left(\lambda_{s}\right), \mathbf{A}=\left(\left(a_{s t}\right)\right), \mathbf{B}=\left(\left(b_{j t}\right)\right), C=\left(c_{s}\right), W=\left(\left(w_{s n t}\right)\right)$, and $\theta=\left(\theta_{s}\right)$ subject to the constraint (4) on the segment sizes. In an internal analysis, all these parameters are estimated simultaneously given the observed three-way, pick-any/J data. For an external analysis, the product coordinates $B$ can be fixed from another MDS analysis and the remaining parameters estimated. We can form the augmented Lagrangian for the constrained optimization as

$\Psi=\sum_{i=1}^{I} \ln \left[\sum_{s=1}^{S} \lambda_{s} \prod_{n=1}^{N} \prod_{j=1}^{J} \Phi\left(f_{s j n}\right)^{\delta_{i j n}} \cdot\left(1-\Phi\left(f_{s j n}\right)\right)^{1-\delta_{i j n}}\right]-\mu\left(\sum_{s=1}^{S} \lambda_{s}-1\right)$,

where $\mu$ is the Lagrange multiplier and $f_{s j n}$ is now rewritten as

$$
f_{s j n}=\frac{h_{s}^{2}-\sum_{t=1}^{T} v_{n t}^{2}\left(a_{s t}-b_{j t}\right)^{2}}{g_{s}^{2}},
$$

with the squared terms $h_{s}^{2}, v_{n t}^{2}$, and $g_{s}^{2}$, having been substituted for $c_{s}, w_{s n t}$, and $\theta_{s}$, respectively, to ensure nonnegativity of these parameters. Also, if the reparameterized options are invoked, the parameters $\alpha_{k t}$ and $\gamma_{m s}$ are estimated in lieu of $b_{j t}$ and $\gamma_{s}$, respectively. A modified conjugate gradient algorithm is utilized (cf. DeSarbo and Hoffman, 1986) along with rational starting procedures (cf. Böckenholt and Böckenholt, 1991) to estimate the 
model parameters. For the reparameterized model options, the posterior probabilities and product locations can be regressed against the respective consumer and product characteristics at the initial iteration to obtain rational starting values of the parameters $\alpha_{k t}$ and $\gamma_{m s}$ (cf. Wedel and DeSarbo, 1995).

In practice, the value of $S$ and $T$ have to be inferred from the data for an internal analysis. We use the consistent AIC (Bozdogan, 1987) as a heuristic for model selection. ${ }^{2}$ The value of $S$ and $T$ is chosen to minimize CAIC, which is here defined as

$$
\left.C A I C_{S T}=-2 \ln L+d(I \cdot J \cdot N)+1\right),
$$

where $d$ is the effective number of free parameters estimated. The proposed model requires the estimation of $S-1$ independent segment sizes. $2 S$ threshold and variance parameters, $S T$ ideal-point coordinates, $J T$ product coordinates, and $T N$ dimensional weights. ${ }^{3}$ Although model identification is not an issue for the majority of exponential family mixtures (see Titterington, Smith, and Makov, 1985; Titterington, 1990), it is important to note that the proposed spatial interaction model is identified only for $S \geq T$. Following De Soete and Winsberg (1993), the appropriate number of segments, $S$, is first determined by comparing the CAIC heuristics of models with different number of scgments and maximum dimensionality. Once the appropriate number of segments is determined, the appropriate dimensionality, $T(T \leq S)$, is identified by comparing the CAIC heuristics of models with the selected number of segments, but with different dimensionalities. ${ }^{4}$

\section{A commercial application}

We illustrate the proposed model by using the actual commercial data from the technological options reasearch study mentioned in the introduction section. Some twenty-three advanced new product options were under consideration as shown in Table 1. Given the innovative and novel nature of these new product options, and since most consumers had no prior familiarity with the vast majority of them, small group interviews were employed for data collection. Respondents were familiarized with the new options via brief video clips (randomized on laser disk) displaying the nature, function, and specific benefits of each option. Independent judges reviewed each video clip to assure objectivity and clarity. In addition, color booklets containing photographs of each new product option, a description of what each option does, the specific benefits provided to the consumer, as well as the particular features and minimum price of each option were reproduced for respondents, for referral at any time during the process. Table 2 provides an example of how each new product option (e.g., visibility lighting) was described in the booklet. In addition, there were detailed color photographs at the top of the page showing each new product option, and where possible, action-oriented pictures of what the new technology does for the consumer relative to current technology.

The questionnaire used for the personal interviews was thoroughly pretested prior to its final utilization. The final study was conducted in six metropolitan locations. Respondents were recruited using a screen initiated by the high-technology firm on the basis of past research whereby respondents had to be responsible for the purchase of their own auto- 
Table 1. New product options and overall desirabilities.

\begin{tabular}{clcc}
\hline Code & \multicolumn{1}{c}{ New Product Option } & Price & $\begin{array}{c}\text { Percentage of Respondents } \\
\text { Choosing Option }\end{array}$ \\
\hline A & Visibility lighting & $\$ 180$ & 71.3 \\
B & Adaptive cruise control & $\$ 500$ & 15.2 \\
C & Collision warning & $\$ 375$ & 31.6 \\
D & Tire pressure management & $\$ 150$ & 50.3 \\
E & Vision enhancement & $\$ 825$ & 26.6 \\
F & Digital cellular phone & $\$ 500$ & 9.0 \\
G & All weather windows & $\$ 75$ & 88.3 \\
H & Fast brake lights & $\$ 25$ & 59.8 \\
I & Adaptive head-up display & $\$ 250$ & 23.1 \\
J & Position location and navigation & $\$ 1500$ & 6.6 \\
K & Anticipatory trouble reporting & $\$ 275+\$ 10 /$ month & 13.0 \\
L & Keyless entry guardian & $\$ 135$ & 51.9 \\
M & Road condition sensors & $\$ 150$ & 34.3 \\
N & Occupant environment & $\$ 375$ & 9.6 \\
O & Voice-activated control & $\$ 400$ & 8.0 \\
P & Perimeter lighting & $\$ 100$ & 38.8 \\
Q & Wide-view mirrors & $\$ 50$ & 78.2 \\
R & Entertainment package & $\$ 650$ & 6.6 \\
S & Light adapting mirrors & $\$ 300$ & 33.2 \\
T & Roadside emergency communicator & $\$ 200+\$ 10 /$ call & 30.3 \\
U & Self-sealing tires & $\$ 35$ extra/tire & 69.4 \\
V & Extended mobility tires & $\$ 400$ extra/car & 24.5 \\
W & Mobile office & $\$ 275$ & 7.2 \\
\hline
\end{tabular}

Table 2. Example of description of new product options.

\section{What It Does}

Visibility lighting is a high-intensity low-beam headlamp that dramatically improves the driver's ability to see the surrounding environment and to be seen by others.

\section{Benefits}

- Visibility lighting provides better visibility of the roadway ahead to help drivers avoid accidents.

- Roadway signs are easier to spot and read.

- Visibility lighting improves your vehicle's ability to be seen by other drivers therefore reducing your chances of being hit.

\section{Features}

- Whiter light

- Long-life headlamp

- Reduced power consumption and improved fuel economy

Price

- $\$ 180$

mobiles, be between twenty-eight and fifty-five years of age, own or lease at least one vehicle less than four years old, and had not participated in a previous research study within the past six months. There were 376 respondents in the final sample. 
A laser disk player displayed each of the twenty-three options in a randomized fashion to minimize potential order effects. After each option was shown on the monitor, the respondents were asked to complete a few monadic questions which followed for that particular option. This process continued until all twenty-three options had been shown. The color booklet displaying the options was distributed just prior to showing the video clips. Respondents were asked to then select as many or as few of these options that they would buy (minimum price levels were displayed so as to obtain an upper bound for demand) on their next automotive purchase. These responses constituted the pick-any/J data $(J=23)$ that were analyzed using the proposed model. Unfortunately, we are unable to discuss all the additional data collected due to the recency and highly confidential nature of the research study; the client firm permitted us to report only analyses based on the two-way, pick-any/J data without the reparameterized model options.

\subsection{Results from an internal analysis}

The pick-any/J data were subject to internal analysis using the proposed approach. The model selection process discussed in the previous section revealed the presence of three segments, and the CAIC pointed to a two-dimensional solution for these three segments. The derived joint space map is shown in Figure 1. The product locations are somewhat dispersed over the joint space. The segment locations, however, are all located in the southwest quadrant. The respective arcs for these segments indicate the boundary of the threshold region for each segment. Hence, the three segments are almost nested within each other, at least in terms of their respective bundle compositions. Thus, segment 1 constitutes 45 percent of the sample and desires a bundle composed of options $A, G, H, Q$ and $U$. Segment 2 constitutes 35 percent of the sample and shows preference for a larger bundle. In addition to the options desired by segment 1, segment 2 also prefers options D, L, M, P, and S. Finally, segment 3 constitutes the remaining 20 percent of the sample and exhibits the largest bundle composition that includes options B, C, E, I, T, and V, in addition to the bundle composition of segment 2 (and thus segment 1). Hence, in the present situation, there appears to be a primary, secondary, and tertiary bundle of options from a market standpoint. ${ }^{5}$ This nesting is congruent with the DX, LX, and EX product lines in many brands of automobiles.

The horizontal dimension of the joint space map appears to reflect the overall popularity (or market share) of the various options, with the most preferred options to the left and the least preferred options to the right. In general, the most preferred options are less expensive than the least preferred options although there are a few exceptions. From Table 1, the most preferred options are visibility lighting (A: 71 percent), tire pressure management (D: 50 percent), all weather windows (G: 88 percent), fast brake lights (H: 60 percent), keyless entry guardian (L: 52 percent), wide view mirrors (Q: 78 percent), and selfsealing tires (U: 69 percent). The least preferred options are position location (J: 6.6 percent), entertainment package ( $R: 6.6$ percent), mobile office (W: 7.2 percent), voice-activated control (O: 8.0 percent), digital cellular phone ( $F$ : 9.0 percent), and occupant environment (N: 9.6 percent). 


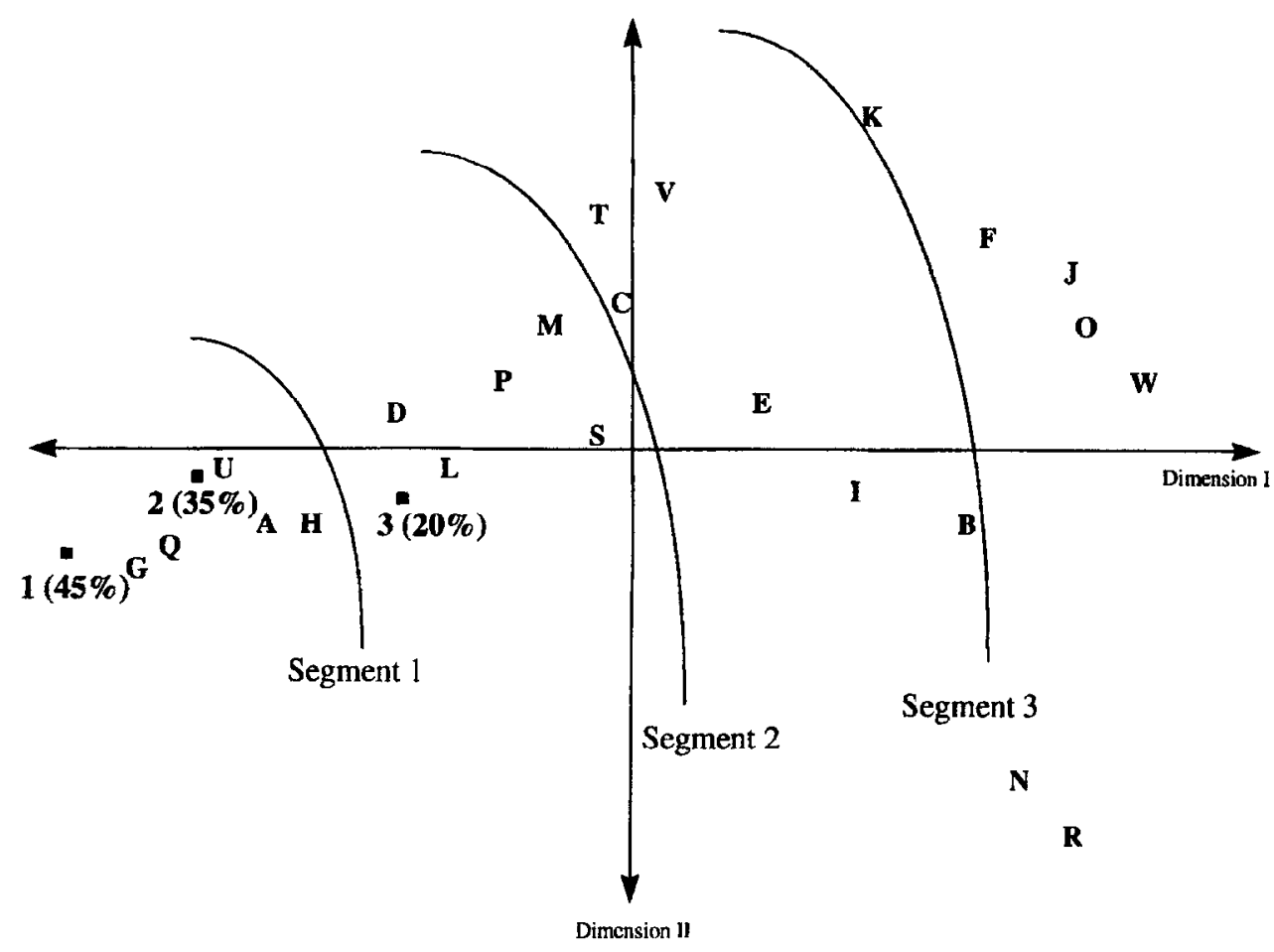

Figure 1. Derived joint space map of technological options and market segments.

The vertical dimension appears to distinguish "safety/emergency" external electronic options (toward the top), versus "comfort/ride" internal electronic options (towards the bottom). Toward the upper portion of the figure, option $\mathrm{K}$ offers the benefit of anticipatory trouble reporting that lessens the possibility of having a catastrophic failure while driving. Option V allows the driver to make a safe passage to the nearest service station after a total loss of air pressure. Option $T$ provides a quick dispatch of the nearest roadside assistance. Option F, while allowing better communication by virtue of digital technology, offers the basic benefit of calling for assistance over a long range when stranded. In contrast, towards the lower portion of the figure, option $\mathrm{N}$ provides an advanced occupant environment where each passenger can configure their seating and climate control to their own individual liking. Option $\mathbf{R}$ provides individualized audio and video entertainment with power for electrical items brought into the automobile.

Table 3 presents the predicted probabilities of choosing the twenty-three options within each of the three derived segments. The estimated parameters were substituted into expression (2) to compute these choice probabilities for each segment. The options have been rearranged according to the bundle compositions corresponding to the three segments; the 
Table 3. Predicted probabilities of choice for each derived segment.

\begin{tabular}{clccc}
\hline Code & New Product Options & Segment I & Segment II & Segment III \\
\hline A & Visibility lighting @180 & 0.56 & 0.71 & 0.87 \\
G & All weather windows @75 & 0.58 & 0.70 & 0.83 \\
H & Fast brake lights @25 & 0.55 & 0.70 & 0.88 \\
Q & Wide-view mirrors \$50 & 0.58 & 0.70 & 0.84 \\
U & Self-sealing tires @35/tire & 0.57 & 0.69 & 0.85 \\
D & Tire pressure management @150 & 0.46 & 0.65 & 0.88 \\
L & Keyless entry guardian @135 & 0.47 & 0.66 & 0.89 \\
P & Perimeter lighting @100 & 0.36 & 0.59 & 0.86 \\
M & Road condition sensors @150 & 0.28 & 0.52 & 0.82 \\
S & Light adapting mirrors @300 & 0.27 & 0.52 & 0.84 \\
C & Collision warning @375 & 0.23 & 0.48 & 0.80 \\
T & Roadside emergency @200 & 0.21 & 0.45 & 0.75 \\
E & Vision enhancement @825 & 0.16 & 0.40 & 0.74 \\
I & Adaptive head-up display @250 & 0.12 & 0.34 & 0.69 \\
V & Extended mobility tires @400 & 0.15 & 0.38 & 0.68 \\
B & Adaptive cruise control @500 & 0.05 & 0.22 & 0.52 \\
K & Anticipatory trouble reporting @275 & 0.04 & 0.20 & 0.41 \\
F & Digital cellular phone @500 & 0.01 & 0.11 & 0.28 \\
J & Position location and navigation @1500 & 0.01 & 0.08 & 0.20 \\
N & Occupant environment @375 & 0.02 & 0.11 & 0.21 \\
O & Voice-activated control @400 & 0.01 & 0.10 & 0.25 \\
R & Entertainment package @650 & 0.01 & 0.07 & 0.11 \\
W & Mobile office @275 & 0.01 & 0.09 & 0.25 \\
\hline
\end{tabular}

fourth and last group of options do not meet the threshold for any of the segments and are the least preferred over all segments. From Table 3, note that the likelihood of choosing each option increases across the derived segments. The estimated threshold parameters $\left(c_{s}\right)$ also increase across the three segments with values of $1.1,1.9$, and 2.1 respectively. Given the proximity of the ideal point locations for the three segments, the number options in the bundle composition for segment I is the smallest. The bundle composition for segment I is nested within that of segment II, which in turn is nested within that of segment III.

The common (primary) bundle across the three segments is composed of visibility lighting $(A)$, all weather windows $(G)$, fast brake lights $(H)$, wide view windows $(Q)$, and selfsealing tires $(\mathrm{U})$. These options appeared to offer the most functional value for a total additional price of $\$ 470$. The next bundle is more expensive with a total additional price of $\$ 835$ but clearly did not offer incremental value to segment 1 for consumers in this segment to pay the extra price. The third bundle appears to be more of a high-end bundle with a total additional price of $\$ 2,550$. This bundle appeals to Segment 3 , which seems to consist of consumers who find several of the new product options to be attractive.

Respondents were also asked whether they believed each option should be included as standard equipment on the vehicle, given that it will add the price shown to the cost of the vehicle to the consumer. We indicate in Table 4 the bundle-specific average of the percentage of respondents answering yes for each option in a particular bundle, for each of the three segments. 
Table 4. The bundle-specific average of the percentage of respondents answering yes for each option in bundle.

\begin{tabular}{lccc}
\hline & Segment I & Segment II & Segment III \\
\hline Primary bundle & 0.68 & 0.75 & 0.80 \\
Secondary bundle & 0.28 & 0.45 & 0.58 \\
Tertiary bundle & 0.19 & 0.25 & 0.40 \\
\hline
\end{tabular}

For the primary bundle, segment 1 has a smaller percentage of respondents who feel that the bundle of options, on average, should be standard. This segment appears to be the most price sensitive of the derived segments. Segment 2 appears to be somewhat less price sensitive than segment 1 . Finally, consumers in segment 3 are willing to pay for the larger bundle as standard equipment. Consumers in segment 3 appear to be the least price sensitive. Note, also, that within any of the segments, the bundle-specific choice averages decrease, as should be expected.

Moorthy (1984) has discussed the advantages of benefit segmentation based on consumer self-selection. In the present case, it is relatively more important to know the alternative bundle compositions desired by different segments, and the relative sizes and compositions of the segments, although it would appear that the derived segments are also likely to differ in terms of their preference for different types of vehicles, previous vehicle ownership, driving habits, and so on. Unfortunately, we are not at liberty to discuss these additional analyses.

\subsection{Comparison with a traditional approach}

As mentioned in the introduction section, correspondence analysis followed by some form of cluster analysis is fraught with methodological difficulties. To examine if such a traditional two-step procedure would render similar results to that obtained with the proposed latent class MDS procedure, we performed such a traditional analysis with the same data from our empirical application. In order to keep the same basis for comparison, we constrained the analysis to two dimensions and three clusters. We obtained a two-dimensional configuration of the twenty-three technological options (correspondence analysis) and average locations (following K-MEANS cluster analysis) for the three clusters of respondents. A canonical correlation analysis was utilized to calibrate the degree of congruence between the configurations produced by this two-step approach with that of the proposed procedure, given the rotational indeterminacies involved in each of these respective solutions. For the twenty-three technological options space, the two canonical correlations were 0.967 and 0.656 suggesting one dimension in common.

The class/cluster memberships were then compared by calculating phi coefficients between the dummy variable class/cluster membership matrices to investigate the need for any permutation of class/cluster labels to maximize congruence. The maximum phi coefficients were $0.292,0.196$, and 0.038 , suggesting very little congruence between the two classifications. After such interchanges, we then computed the proportion of similar classifications in the two grouping schemes. Only $40.9 \%$ of the sample was categorized in the same groupings (chance alone would suggest that $33.3 \%$ could be categorized correctly). 
This, together with the results of the canonical correlation analysis, suggest that these two procedures render quite different results for this application-a finding we would expect to encounter in general given the problematic nature of the two-step approach as discussed in the introduction. ${ }^{6}$

\section{Conclusion}

We have presented a spatial interaction model for simultaneously segmenting consumers and estimating a joint map of product options and segment ideal points that describes the respective bundle compositions for each segment. The model also estimates the probability of segment membership for each consumer in the sample, and if additional product or background respondent data are available, allows for direct reparameterization of the segment memberships as well as product locations to facilitate further managerial interpretation and aid in targeting segments. The proposed approach can accommodate threeway data collected over different scenarios and usage situations, and allows for differential weighting of the dimensions for each scenario/situation within each segment.

Apart from survey data involving consumer judgments, the proposed approach can be gainfully utilized to analyze consumer purchase behavior. For instance, one can analyze market-basked scanner data to investigate complementarity among different types of products over time. Or with various household inventory/diary records, the procedure could be gainfully employed to structurally represent the joint purchase of various brands within the same product class (e.g., brands of breakfast cereals) over time. Alternatively, the proposed methodology could be utilized in the examination of choice or purchase shifts that occur as a function of various experimental treatments (e.g., to examine how choices differ as prices are experimentally manipulated). Finally, the proposed procedure could be easily modified to spatially represent the results of choice based conjoint studies.

\section{Notes}

1. Note that the assumption of conditional indepcndence implies that the choices are independent $g$ iven the structural part of the model. The structural part of the model actually captures the covariances between the choices arising from the compositional pattern of the bundles.

2. Testing the null hypothesis of $S$ segments versus the alternative of $S+1$ segments, or the null hypothesis of $T$ dimensions against the alternative of $T+1$ dimensions, cannot however be carried out since the usual likelihood ratio tests are not asymptotically distributed as chi-square (Aitkin and Rubin, 1985) given the regularity conditions may not theoretically hold.

3. The effective number of degrees of freedom (d.f.) for the full model is affected by centering and scale indeterminacies (a loss of $2 T$ d.f.). In two-way analyses, there are also rotational indeterminacies (a loss of $T(T+1) / 2$ d.f. $)$.

4. Note that there is no confounding between the number of segments and the number of dimensions because the saturated model for any number of segments is in fact identical to a latent class model with alternativespecific constants, and that model is restricted by constraining the number of dimensions.

5. Note that the nesting is not a necessary outcome of the model but of the specific application. In general, each segment can desire a distinct bundle.

6. DeSarbo, Wedel, Vriens, and Ramaswamy (1992) find similar results with respect to their latent class metric conjoint procedure. 


\section{References}

Aitkin, M., and D.B. Rubin. (1985). "Estimation and Hypothesis Testing in Finite Mixture Models." Journal of the Royal Statistical Society, B47, 67-75.

Böckenholt, U., and I. Böckenholt. (1991). "Constrained Latent Class Analysis: Simultaneous Classification and Scaling of Discrete Choice Data." Psychometrika 56, 699-716.

Böckenholt, U., and W. Gaul. (1986). "Analysis of Choice Behavior via Probabilistic Ideal Point and Vector Models." Applied Stochastic Models and Data Analysis 2, 202-226.

Bozdogan, H. (1987). "Model Selection and Akaike's Information Criterion (AIC): The General Theory and Its Analytical Extensions." Psychometrika 52, 345-370.

Dayton, M.C., and G.B. MacReady. (1988). "Concomitant Variable Latent Class Models." Journal of the American Statistical Association 83, 401, 173-179.

DeSarbo, W.S., and J.D. Carroll. (1985). "Three-Way Metric Unfolding via Alternating Weighted Least Squares." Psychometrika 50(3), 275-300.

DeSarbo, W.S., and D.L. Hoffman. (1987). "Constructing MDS Joint Spaces from Binary Choice Data: A New Multidimensional Unfolding Threshold Model for Marketing Research." Journal of Marketing Research 24 (February), 40-54.

DeSarbo, W.S., D. Lehman, S. Gupta, M. Holbrook, and W. Havlena. (1985). "A Three-Way Unfolding Methodology for Asymmetric Binary Proximity Data." Applied Psychological Measurement 11, 397-418.

DeSarbo, W.S., A.K. Manrai, and L.A. Manrai. (1994). "Latent Class Multidimensional Scaling: A Review of Recent Developments in the Marketing and Psychometric Literature." Richard P. Bagozzi (ed.), in Advanced Methods of Marketing Research pp. 190-222, Cambridge, MA: Blackwell.

DeSarbo, W.S., and V. Ramaswamy. (1994). "CRISP: Customer Response Based Iterative Segmentation Procedures for Response Modeling in Direct Marketing." Journal of Direct Marketing 8(3) (Summer), 7-20.

DeSarbo, W.S., V. Ramaswamy, and P. Lenk. (1993). "A Latent Class Procedure for the Structural Analysis of Two-way Compositional Data." Journal of Classification. 10, 159-193.

DeSarbo, W.S., and V.R. Rao. (1986). “A Constrained Unfolding Methodology for Product Positioning." Marketing Science 5 (Winter), 1-19.

DeSarbo, W.S., M. Wedel, M. Vriens, and V. Ramaswamy. (1992). "Latent Class Metric Conjoint Analysis." Marketing Letters 3, 273-288.

De Soete, G., and W.S. DeSarbo. (1991). "A Latent Class Probit Model for Analyzing Pick-Any/N Data." Journal of Classification 8, 45-64.

De Soete, G., and S. Winsberg. (1993). "A Latent Class Vector Model for Preference Ratings." Journal of Classification 10(2), 195-218.

Green, P.E., C.M. Schaffer, and K.M. Patterson. (1988). "A Reduced-Space Approach to the Clustering of Categorical Data in Market Segmentation." Journal of the Market Reserach Society 30(3), 267-288.

Hoffman, D.L., and G.R. Franke. (1986). "Correspondence Analysis: Graphical Representation of Categorical Data in Marketing Research." Journal of Marketing Research 23 (August), 213-227.

Holbrook, M.B., W.L. Moore, and R.S. Winer. (1982). "Constructing Joint Spaces from Pick-any Data: A New Tool for Consumer Analysis." Journal of Consumer Research 9(1), 99-105.

Jedidi, K., and W.S. DeSarbo. (1991). "A Stochastic Multidimensional Scaling Procedure for the Spatial Representation of Three-Mode, Three-Way, Pick Any/J Data." Psychometrika 56(3), 471-494.

Kamakura, W., M. Wedel, and J. Agarwal. (1994). "Concomitant Variable Latent Class Models for Conjoint Analysis." International Journal of Research in Marketing 11(5), 451-464.

Lebart, L., A. Morineau, and K.M. Warwick. (1984). Multivariate Descriptive Statistical Analysis: Correspondence Analysis and Related Techniques for Large Matrices. New York: John Wiley.

McFadden, D. (1976). "Quantal Choice Analysis: A Survey." Annals of Economic and Social Measurement 5 (Fall), 363-390.

Moorthy, S. (1984). "Market Segmentation, Self-Selection, and Product Line Design." Marketing Science 3 (Fall), 288-307.

Nishisato, S. (1980). Analysis of Categorical Data: Dual Scaling and its Applications. Toronto: University of Toronto Press. 
Stefflre, V. (1971). New Products and New Enterprises: A Report on an Experiment in Applied Social Science. Irvine: School of Social Science, University of California.

Takane, Y. (1983). "Choice Model Analysis of the 'Pick-Any/N' Type of Binary Data." Paper presented at the European Psychometric and Classification Meetings, Jouy-en-Josas, France, July.

Titterington, D.M. (1990). "Some Recent Research in the Analysis of Mixture Distributions." Statistics 4, 619-641. Titterington, D.M., A.F.M. Smith, and U.E. Makov. (1985). Statistical Analysis of Finite Mixture Distributions. New York: John Wiley.

Wedel, M., and W.S. DeSarbo. (1995). "A General Latent Class Stochastic MDS Methodology for Simultaneous Segmentation and Positioning." Research Report 94311, Graduate School/Research Institute, University of Groningen, Netherlands.

Wind, Y. (1978). "Issues and Advances in Segmentation Research." Journal of Marketing Research 15 (August), 317-337. 